\title{
The Primitive Permutation Groups of Degree Less Than 4096
}

\author{
Hannah J. Coutts, Martyn Quick, Colva M. Roney-Dougal \\ School of Mathematics and Statistics, University of St Andrews, Fife, KY16 9SS, Scotland
}

\begin{abstract}
In this paper we use the Classification of the Finite Simple Groups, the O'NanScott Theorem and Aschbacher's theorem to classify the primitive permutation groups of degree less than 4096. The results will be added to the primitive groups databases of GAP and MAGMA.
\end{abstract}

\section{Introduction and history}

The study of primitive permutation groups has a long and rich history. The earliest reference to primitive groups is in the work of Ruffini in 1799 where he divided non-cyclic permutation groups into intransitive, imprimitive and primitive cases while trying (unsuccessfully) to prove the insolubility of the general quintic equation. In 1871 Jordan [15] made one of the first attempts at classifying the primitive groups up to degree 17 , one of his many significant achievements relating to primitive groups. Some of Jordan's enumerations were later corrected by Cole [5] and Miller [24, 25, 26, 27, 28, 29, 30] in the last years of the 19th century. The work of Martin [23] in 1901 and Bennett [2] in 1912 completed the classification up to degree 20.

At this stage the lists of groups were getting too big to work with by hand without a high chance of error and little significant progress was made until the birth of symbolic computation in the 1960s. Sims [35] classified all the primitive groups up to degree 50 as well as correcting the exisiting classifications. The full lists were never published but were available to the mathematical community and eventually formed one of the earliest databases in computational group theory. Further important developments were made following the announcement of the Classification of the Finite Simple Groups. In 1988 Dixon and Mortimer [7] used the O'Nan-Scott Theorem to classify all non-affine primitive permutation groups of degree less than 1000: the numbers of affine groups of these degrees are too large to be handled without computers. These groups were made into a database in GAP by Thießen [37] together with the soluble affine type groups of degree less than 255, which were classified by Short [34].

Email addresses: $\{$ hcoutts, martyn, colva\}@mcs.st-andrews.ac.uk 
More recently Eick and Höfling [9] classified all soluble affine groups of degree less than 6561 and Roney-Dougal and Unger [33] classified all affine groups of degree less than 1000. In 2005 Roney-Dougal [32] classified all primitive permutation groups of degree less than 2500 , simultaneously checking and correcting the existing results. As a consequence we shall only consider primitive permutation groups of degree greater than 2499 .

This paper extends the classification of the primitive permutation groups up to degree 4095, using the framework of the O'Nan-Scott Theorem and with Aschbacher's theorem and CFSG as important tools. Section 2 sets out some notation and basic ideas that will be needed throughout the paper. The following sections treat each O'Nan-Scott class in turn, explaining in detail how the primitive groups are found. For some of the classes it is possible to automate almost all of the process while for others it is necessary to perform some theoretical calculations to reduce the computational burden. Section 7 discusses the methods used to ensure accuracy in computation and presentation and the final section contains the tables of primitive groups. The groups will be added to the databases of GAP [10] and MAGMA [4].

\section{Preliminaries and notation}

We begin by setting up some notation and stating a few results which will be needed later in the paper. A useful reference for more details is [8].

Throughout all groups are finite and $d$ denotes the degree of a permutation group, $n$ is a non-zero positive integer, $p$ is a prime and $q$ is a prime power. We use the notation $\alpha^{g}$ to denote the action of a group element $g$ on $\alpha$. A group $G$ acting on a set $\Omega$ is transitive if for all $\alpha, \beta$ in $\Omega$ there exists some $g \in G$ such that $\alpha^{g}=\beta$. A subset $\Delta$ of $\Omega$ is a block for $G$ if for all $g \in G$ either

$$
\Delta^{g}=\Delta \text { or } \Delta^{g} \cap \Delta=\emptyset .
$$

The action of $G$ is primitive if it is transitive and all blocks are trivial, that is either $|\Delta|=1$ or $\Delta=\Omega$. A group which is not transitive is intransitive and a transitive group which preserves a non-trivial block is imprimitive.

The socle of a group $G$ is the subgroup generated by its minimal normal subgroups. If $G$ is primitive then $\operatorname{Soc}(G)$ is isomorphic to the direct product of one or more copies of a simple group $T$. A group $G$ is almost simple if

$$
T \cong \operatorname{Inn}(T) \unlhd G \leq \operatorname{Aut}(T)
$$

for some nonabelian simple group $T$.

Theorem 1 (O'Nan-Scott Theorem). Let $G$ be a primitive permutation group of degree $d$, and let $H:=\operatorname{Soc}(G) \cong T^{m}$ with $m \geq 1$. Then one of the following holds.

1. $H$ is regular and 
(a) $T$ is cyclic of order $p$ and $|H|=p^{m}$. Then $d=p^{m}$ and $G$ is isomorphic to a subgroup of the affine general linear group $\mathrm{AGL}_{m}(p)$. We call $G$ an "affine type" group.

(b) $m \geq 6$, the group $T$ is non-abelian and $G$ is a group of "twisted wreath product type", with $d=|T|^{m}$.

2. $H$ is non-regular and non-abelian and

(a) $m=1$ and $G$ is almost simple.

(b) $m \geq 2$ and $G$ is permutation isomorphic to a subgroup of the product action wreath product $P\left\{\mathrm{~S}_{m / l}\right.$ of degree $d=n^{m / l}$. The group $P$ is primitive of type 2.(a) or 2.(c) of degree $n$ and $\operatorname{Soc}(P) \cong T^{l}$.

(c) $m \geq 2$ and $G$ is a group of "diagonal type" with $d=|T|^{m-1}$.

We can see immediately that there are no twisted wreath product type groups of degree less than 4096 and so this class is not considered further. We examine the other cases in the order above.

Let $G$ be an almost simple classical group, and assume that if $G$ contains a graph automorphism then $\operatorname{Soc}(G) \notin\left\{\mathrm{S}_{4}\left(2^{i}\right), \mathrm{P} \Omega_{8}^{+}(q)\right\}$. Then Aschbacher's theorem [1] states that any subgroup of $G$ lies in one of nine Aschbacher classes $\mathcal{C}_{i}$ for $i \in\{1, \ldots, 9\}$. Each $\mathcal{C}_{i}$ is described in detail in [16]. A group is $A S$ maximal if it is the stabilizer in the classical group of the geometry associated with the Aschbacher class.

In Section 4 heavy use will be made of $\mathcal{C}_{1}$ and so we give a definition of this class. Let $G$ be a classical group acting on the vector space $V$. A subgroup $H$ of $G$ which preserves a proper non-trivial subspace of $V$ is reducible. Otherwise $H$ is irreducible. The Aschbacher class $\mathcal{C}_{1}$ of $G$ consists of all reducible subgroups of $G$. If $H$ is a maximal $\mathcal{C}_{1}$ subgroup of $G$ stabilising $U \leq V$ then the restriction of the form to $U$ is either nondegenerate or identically zero.

We classify groups up to permutation isomorphism, using the fact that subgroups of $\mathrm{S}_{d}$ are permutation isomorphic if and only if they are conjugate in $\mathrm{S}_{d}$. If two maximal subgroups of a group $G$ are conjugate in $\operatorname{Aut}(G)$ then the images of the actions of $G$ on their cosets are permutation isomorphic. The primitive groups are partitioned into cohorts, where two groups are in the same cohort if their socles are permutation isomorphic.

If a (projectively) almost simple group $G$ with socle $T$ has a maximal subgroup $M$ for which $M \cap T$ is a proper, non-maximal subgroup of $T$ then $M$ is a novelty. If $T \leq M$ then $M$ is a triviality and corresponds to a non-faithful action. Otherwise $M$ is an ordinary maximal subgroup. The index of any novelty in $G$ is greater than the index of the largest ordinary maximal subgroup of $G$.

We use the notation of [16] for all groups with a few exceptions. The linear, symplectic and unitary simple groups are denoted $\mathrm{L}_{n}(q), \mathrm{S}_{2 m}(q)$ and $\mathrm{U}_{n}(q)$ respectively. The stabilizer of $\alpha$ under the action of $G$ is denoted by $G_{\alpha}$ and the centre of $G$ by $\mathrm{Z}(G)$. We write $\operatorname{Inn}(G)$ for the inner automorphism group of $G$, and $\operatorname{Out}(G)=\operatorname{Aut}(G) / \operatorname{Inn}(G)$. A group is $C S$ if it has computable subgroups in MaGma V2.14-12.

The following is well known (for example [16, Proposition 2.9.1]). We will treat the following groups as the right hand side of the isomorphism. 
Theorem 2. The following classical groups are not simple: $\mathrm{L}_{2}(q)$ for $q \leq 3$, $\mathrm{P} \Omega_{2}^{ \pm}(q), \mathrm{P} \Omega_{4}^{+}(q), \mathrm{U}_{3}(2)$. With the exception of these and (possibly isomorphic) groups given below, all groups $\mathrm{L}_{n}(q), \mathrm{S}_{2 m}(q), \mathrm{U}_{n}(q)$ and $\mathrm{P} \Omega_{n}^{\epsilon}(q)$ (where $\epsilon \epsilon$ $\{+,-, \circ\})$ are simple. Furthermore, the list below includes all isomorphisms between pairs of classical or alternating groups.
$\mathrm{L}_{2}(4) \cong \mathrm{L}_{2}(5) \cong \mathrm{A}_{5}$
$\mathrm{L}_{3}(2) \cong \mathrm{L}_{2}(7)$
$\mathrm{L}_{2}(9) \cong \mathrm{S}_{4}(2)^{\prime} \cong \mathrm{A}_{6}$
$\mathrm{L}_{4}(2) \cong \mathrm{A}_{8}$
$\mathrm{U}_{2}(q) \cong \mathrm{S}_{2}(q)=\mathrm{L}_{2}(q)$
$\mathrm{U}_{4}(2) \cong \mathrm{S}_{4}(3)$
$\mathrm{P} \Omega_{2 m+1}\left(2^{i}\right) \cong \mathrm{S}_{2 m}\left(2^{i}\right), i \geq 1$
$\mathrm{P} \Omega_{3}(q) \cong \mathrm{L}_{2}(q), q$ odd
$\mathrm{P} \Omega_{5}(q) \cong \mathrm{S}_{4}(q), q$ odd
$\mathrm{P} \Omega_{4}^{-}(q) \cong \mathrm{L}_{2}\left(q^{2}\right)$
$\mathrm{P} \Omega_{6}^{+}(q) \cong \mathrm{L}_{4}(q)$
$\mathrm{P} \Omega_{6}^{-}(q) \cong \mathrm{U}_{4}(q)$

This completes the notation and preliminary results required. We now treat each O'Nan-Scott class in turn.

\section{Affine type groups}

In this section we classify the primitive permutation groups of affine type of degree $2500 \leq d<4096$. Throughout, let $V=\mathbb{F}_{p}^{k}$ be a vector space.

Definition 3. The affine general linear group $\operatorname{AGL}_{k}(p)$ consists of all functions $f: V \rightarrow V$ given by $f(v)=v a+u$ where $u \in V$ and $a \in \mathrm{GL}_{k}(p)$. The maps with $a=1$ generate $T \unlhd \operatorname{AGL}_{k}(p)$.

The subgroup $T$ is regular and equal to the socle of $\mathrm{AGL}_{k}(p)$. The group $\operatorname{AGL}_{k}(p)$ is a split extension of $T \cong V$ by the stabilizer in $\operatorname{AGL}_{k}(p)$ of $0_{V}$. Thus $\operatorname{AGL}_{k}(p) \cong V: \operatorname{GL}_{k}(p)$.

A primitive group $G$ is of affine type if $G \leq \operatorname{AGL}_{k}(p)$ and $\operatorname{Soc}(G) \cong T$. The action of the normalizer $N=\mathrm{N}_{S_{d}}(T)$ on $T$ is permutation isomorphic to the action of $\operatorname{AGL}_{k}(p)$ on $V$. If a group $G$ such that $T \leq G \leq N$ is primitive then $G_{0_{V}}$ is naturally isomorphic to an irreducible subgroup of $\mathrm{GL}_{k}(p)$. So classifying the primitive permutation groups of affine type of degree $2500 \leq d<4096$ corresponds to classifying the irreducible subgroups of $\mathrm{GL}_{k}(p)$ with $2500 \leq p^{k}=d<$ 4096. Two groups of affine type $T: K_{1}$ and $T: K_{2}$ are permutation isomorphic if and only if the irreducible subgroups $K_{1}, K_{2} \in \mathrm{GL}_{k}(p)$ are conjugate in $\mathrm{GL}_{k}(p)$.

Case $k=1$. In this case, all subgroups $K$ of $\mathbb{F}_{p}^{*}=\mathrm{GL}_{1}(p)$ are irreducible. There is one conjugacy class of affine type groups for each divisor of $p-1$.

Case $k>1$. Here $(k, p) \in\{(2,53),(2,59),(2,61),(5,5)\}$. For the first three of these can directly compute representatives for each conjugacy class of the irreducible subgroups of $\mathrm{GL}_{k}(p)$. The corresponding subgroups of $\mathrm{AGL}_{k}(p)$ are constructed by taking semidirect products of the irreducible subgroups with their natural modules.

The group $\mathrm{GL}_{5}(5)$ is somewhat larger. First we calculate its trivial maximals; in $\mathrm{GL}_{5}(5)$ there is a unique proper subgroup $M:=\mathrm{SL}_{5}(5): 2$ containing $\mathrm{SL}_{5}(5)$. We let $L$ be the union of the class representatives of the non-trivial irreducible maximal subgroups of $\mathrm{GL}_{5}(5), M$ and $\mathrm{SL}_{5}(5)$ : note that these are the maximal subgroups that do not contain $\mathrm{SL}_{5}(5)$. We then let $L_{1}$ be the union of the class representatives of the irreducible subgroups of each member of $L$. Finally, we check $L \cup\left\{\mathrm{GL}_{5}(5), M, \mathrm{SL}_{5}(5)\right\}$ for conjugacy under $\mathrm{GL}_{5}(5)$, and discard any 
duplicates. This method avoids a large enough part of the subgroup lattice to make the computation manageable.

These computational methods serve to prove the following theorem.

Theorem 4. Let $G$ be a primitive permutation group of affine type of degree $2500 \leq d<4096$. If $d$ is prime then $G \cong d: r$ where $r$ divides $d-1$. Otherwise $d=p^{k}$ for $(p, k) \in\left\{53^{2}, 59^{2}, 61^{2}, 5^{5}\right\}$ and $G \cong T: K$ where $T \cong \mathbb{F}_{p}^{k}$ and $K$ is an irreducible subgroup of $\mathrm{GL}_{k}(p)$. The numbers of primitive soluble and insoluble affine groups of non-prime degree $2500 \leq d<4096$ are given in Table 2.

\section{Almost simple groups}

Next we classify the primitive almost simple groups of degree $2500 \leq d<$ 4096. The groups with alternating socle are considered first, followed by the groups with classical, exceptional and finally sporadic socle.

Faithful primitive actions of a group $G$ correspond to conjugacy classes of core-free maximal subgroups of $G$. Hence we can classify the almost simple primitive permutation groups of degree $2500 \leq d<4096$ by finding the maximal subgroups of almost simple groups of index in that range.

We consider the families of simple groups in turn and create a list of groups $T$ which are potential socles of primitive almost simple groups of the correct degrees. Let $P(G)$ denote the smallest $d$ such that $G$ has a faithful primitive permutation action of degree $d$. The following is well known.

Lemma 5. If $G$ is an almost simple group with socle $H$ then $P(G) \geq P(H)$.

\subsection{Alternating and Symmetric groups}

For $d>4$, the groups $\mathrm{A}_{d}$ and $\mathrm{S}_{d}$ in their natural action form a single cohort of improper primitive groups. We do not consider these further.

Proposition 6. Let $G$ be $\mathrm{A}_{n}$ or $\mathrm{S}_{n}$. If $G$ has a faithful non-natural primitive action of degree $2500 \leq d<4096$ then $n \leq 91$. If the point stabilizer $G_{\alpha}$ of this action is transitive on $\{1, \ldots, n\}$ then $G_{\alpha}$ is primitive and $10 \leq n \leq 12$.

Proof. Since $6 !<2500$ we may assume that $n \geq 7$. Let $X$ be a proper subgroup of $\mathrm{A}_{n}$ with $X \neq \mathrm{A}_{n-1}$ and assume that either $X$ is maximal in $\mathrm{A}_{n}$ or else $X=Y \cap \mathrm{A}_{n}$ where $Y$ is a maximal subgroup of $\mathrm{S}_{n}$.

CAse 1: Suppose that $X$ is primitive in its action on $\{1, \ldots, n\}$. By Bochert's theorem [14, Satz 2.4.6]

$$
\left|\mathrm{S}_{n}: X\right|>\lfloor(n+1) / 2\rfloor !
$$

so $\left|\mathrm{S}_{n}: X\right|<8192$ implies $n<15$. We use Magma to find the indices of the primitive maximal subgroups of $\mathrm{A}_{n}$ and $\mathrm{S}_{n}$ for $7 \leq n \leq 14$. Only the groups $\mathrm{A}_{10}, \mathrm{~A}_{11}, \mathrm{~A}_{12}$ and $\mathrm{S}_{10}$ have primitive maximal subgroups of index in the range $2500 \leq d<4096$; each has one conjugacy class of such subgroups of index 
2520. Note that in fact the point stabiliser in this action of $\mathrm{S}_{10}$ has primitive intersection with $\mathrm{A}_{10}$ (in the natural action).

CASE 2: Suppose that $X$ is imprimitive on $\{1, \ldots, n\}$. Let $k$ be the size of some non-trivial block for $X$, so that $1<k<n$, and set $m:=n / k$. We have seen in case 1 that there is no maximal primitive $Y \leq \mathrm{S}_{n}$ such that $X=Y \cap \mathrm{A}_{n}$ is imprimitive. Therefore without loss of generality $X$ is an index 2 subgroup of $\mathrm{S}_{k} \prec \mathrm{S}_{m}$ and $|X|=(k !)^{m}(m !) / 2$. Hence

$$
\left|\mathrm{A}_{n}: X\right|=\left|\mathrm{S}_{n}: Y\right|=(m k) ! /(k !)^{m} m !=f(m, k) .
$$

The function $f(m, k)$ increases monotonically in both variables and the reader may check that for $(m, k) \in\{(2,7),(3,3),(4,2),(5,2)\}$ the value of $f(m, k)$ is less than 2500 , whilst for $(m, k) \in\{(2,8),(3,4),(4,3),(5,3),(6,2)\}$ the value of $f(m, k)$ is greater than 4095 . Hence there is no $n$ such that $\mathrm{A}_{n}$ or $\mathrm{S}_{n}$ has an imprimitive maximal subgroup of index $2500 \leq d<4096$.

CASE 3: Finally, suppose that $X$ acts intransitively on $\{1, \ldots, n\}$. By $[32$, proof of Prop. 4.2] the group $X$ has no orbit of length 1. Let $\Gamma$ be the smallest orbit of $X$ and set $k:=|\Gamma| \leq n / 2$. Then $X \leq\left(\mathrm{S}_{k} \times \mathrm{S}_{n-k}\right) \cap \mathrm{A}_{n}$, so

$$
|X| \leq k !(n-k) ! / 2
$$

and

$$
\left|\mathrm{A}_{n}: X\right|=\left|\mathrm{S}_{n}: Y\right| \geq n ! / k !(n-k) !=\left(\begin{array}{l}
n \\
k
\end{array}\right) \geq\left(\begin{array}{l}
n \\
2
\end{array}\right) .
$$

The upper bound $\left|\mathrm{A}_{n}: X\right|<4096$ implies that $n<92$.

The intransitive maximal subgroups of $\mathrm{A}_{n}$ and $\mathrm{S}_{n}$ are well understood.

Theorem 7. Let $G$ be a primitive almost simple group of degree $2500 \leq d<$ 4096 with socle $\mathrm{A}_{n}$. Then $G$ appears in Table 3.

\subsection{Classical groups}

Recall that a simple classical group takes one of the following forms: linear, $\mathrm{L}_{n}(q)$; symplectic, $\mathrm{S}_{2 m}(q)$; unitary, $\mathrm{U}_{n}(q)$; orthogonal in odd dimension, $\mathrm{P} \Omega_{2 m+1}(q)$ and orthogonal in even dimension, $\mathrm{P} \Omega_{2 m}^{\epsilon}(q)$ with $\epsilon \in\{+,-\}$. Using [16] to find $P\left(\mathrm{Cl}_{n}(q)\right)$ where $\mathrm{Cl}_{n}(q)$ is a simple classical group, we determine the maximum values of $n$ and $q$ such that $P\left(\mathrm{Cl}_{n}(q)\right)<4096$. 


\begin{tabular}{|c|c|c|c|}
\hline Group & $n$ & $q$ & Non-CS \\
\hline $\mathrm{L}_{n}(q)$ & $\begin{array}{l}n=2 \\
n=3 \\
n=4 \\
n=5 \\
n=6 \\
n=7 \\
n=8 \\
9 \leq n \leq 12\end{array}$ & $\begin{array}{l}q \leq 4093 \\
q \leq 61 \\
q \leq 13 \\
q \leq 7 \\
q \leq 5 \\
q \leq 3 \\
q \leq 3 \\
q=2\end{array}$ & $\begin{array}{l}\mathrm{L}_{6}(4), \mathrm{L}_{6}(5) \\
\mathrm{L}_{8}(3)\end{array}$ \\
\hline $\mathrm{S}_{2 m}(q)$ & $\begin{array}{l}m=2 \\
m=3 \\
m=4 \\
5 \leq m \leq 6\end{array}$ & $\begin{array}{l}q \leq 13 \\
q \leq 5 \\
q \leq 3 \\
q=2\end{array}$ & $\begin{array}{l}\mathrm{S}_{6}(4), \mathrm{S}_{6}(5) \\
\mathrm{S}_{8}(3) \\
\mathrm{S}_{12}(2) \\
\end{array}$ \\
\hline $\mathrm{U}_{n}(q)$ & $\begin{array}{l}n=3 \\
n=4 \\
n=5 \\
6 \leq n \leq 7\end{array}$ & $\begin{array}{l}q \leq 13 \\
q \leq 7 \\
q \leq 3 \\
q=2\end{array}$ & $\begin{array}{l}\mathrm{U}_{5}(3) \\
\mathrm{U}_{7}(2)\end{array}$ \\
\hline $\mathrm{P} \Omega_{2 m+1}(q)$ & $\begin{array}{l}m=3 \\
m=4\end{array}$ & $\begin{array}{l}q \leq 5 \\
q=3\end{array}$ & $\begin{array}{l}\mathrm{P} \Omega_{7}(5) \\
\mathrm{P} \Omega_{9}(3)\end{array}$ \\
\hline $\mathrm{P} \Omega_{2 m}^{+}(q)$ & $\begin{array}{l}m=4 \\
5 \leq m \leq 6\end{array}$ & $\begin{array}{l}q \leq 3 \\
q=2\end{array}$ & $\begin{array}{l}\mathrm{P} \Omega_{8}^{+}(3) \\
\mathrm{P} \Omega_{12}^{+}(2)\end{array}$ \\
\hline $\mathrm{P} \Omega_{2 m}^{-}(q)$ & $\begin{array}{l}m=4 \\
5 \leq m \leq 6\end{array}$ & $\begin{array}{l}q \leq 3 \\
q=2\end{array}$ & $P \Omega_{12}^{-}(2)$ \\
\hline
\end{tabular}

Lemma 8. Let $G$ be an almost simple classical group with $P(G)<4096$. Then the socle $H$ of $G$ appears in Table 1.

Proof. By Lemma 5 it suffices to consider the simple classical groups. The formulae for $P(H)$ are given in $[3,16]$ and are all monotonically increasing in each variable.

Linear. The groups $\mathrm{L}_{2}(q)$ for $q \leq 5$ are either soluble or have already been considered by Theorem 2. If $q \notin\{(2, q): q$ odd, $7 \leq q \leq 11\} \cup\{(4,2)\}$ then the minimal degree of a non-trivial permutation representation of $\mathrm{L}_{n}(q)$ is $\left(q^{n}-\right.$ $1) /(q-1)$. All of the exceptions are groups of order less than 4095 apart from $\mathrm{L}_{4}(2) \cong \mathrm{A}_{8}$ which has already been considered. Hence the largest values of $n$ and $q$ for which $P\left(\mathrm{~L}_{n}(q)\right)<4096$ are as given in Table 1 .

Symplectic. We assume that $m>1$ and $(m, q) \neq(2,2)$ by Theorem 2 . The minimal degree of a non-trivial permutation representation of $\mathrm{S}_{2 m}(2)$ is $2^{m-1}\left(2^{m}-1\right)$ for $m \geq 3$. With the exception of $P\left(\mathrm{~S}_{4}(3)\right)=27$, if $m \geq 2$ and $q \geq 3$ then $P\left(\mathrm{~S}_{2 m}(q)\right)=\left(q^{2 m}-1\right) /(q-1)$. 
Unitary. We assume that $n>2$ and $(n, q) \notin\{(3,2),(4,2)\}$ by Theorem 2. If $q \neq 2,5$ then $P\left(\mathrm{U}_{3}(q)\right)=q^{3}+1$, whilst $P\left(\mathrm{U}_{3}(5)\right)=50$. If $q \neq 2$ then $P\left(\mathrm{U}_{4}(q)\right)=$ $q^{4}+q^{3}+q+1$. Now let $n \geq 5$. When $n$ is even $P\left(\mathrm{U}_{n}(2)\right)=2^{n-1}\left(2^{n}-1\right) / 3$. Otherwise

$$
P\left(\mathrm{U}_{n}(q)\right)=\left(q^{n}-(-1)^{n}\right)\left(q^{n-1}-(-1)^{n-1}\right) /\left(q^{2}-1\right) .
$$

Orthogonal, odd dimension. We assume that $m \geq 3$ and $q$ is odd by Theorem 2. Then $P\left(\mathrm{P} \Omega_{2 m+1}(3)\right)=3^{m}\left(3^{m}-1\right) / 2$ and $P\left(\mathrm{P} \Omega_{2 m+1}(q)\right)=\left(q^{2 m}-\right.$ $1) /(q-1)$ for $q \geq 5$.

Orthogonal, plus and minus types. We assume that $m \geq 4$ by Theorem 2 . Then $P\left(\mathrm{P} \Omega_{2 m}^{+}(2)\right)=2^{m-1}\left(2^{m}-1\right)$ and $P\left(\mathrm{P} \Omega_{2 m}^{+}(3)\right)=3^{m-1}\left(3^{m}-1\right) / 2$. For $q \geq 4$ and $\epsilon=+$, or for all $q$ and $\epsilon=-$

$$
P\left(\mathrm{P} \Omega_{2 m}^{\epsilon}(q)\right)=\left(q^{m}-\epsilon\right)\left(q^{m-1}+\epsilon\right) /(q-1) .
$$

In general, the primitive almost simple groups with CS socles can be created computationally, by constructing their maximal subgroups. The group $\mathrm{L}_{12}(2)$ is CS but is extremely large, so will be dealt with in Lemma 14 .

\subsubsection{Reduction of actions}

For some of the remaining classical groups $G$, we shall show that all irreducible subgroups have index $>4095$. We use $[12,13]$ to construct maximal reducible subgroups of $G$.

Proposition 9. Let $H$ be one of the following classical simple groups

$$
\mathrm{L}_{6}(4), \mathrm{L}_{6}(5), \mathrm{L}_{8}(3), \mathrm{S}_{6}(5), \mathrm{S}_{8}(3), \mathrm{U}_{5}(3), \mathrm{U}_{7}(2), \mathrm{P} \Omega_{9}(3), \mathrm{P} \Omega_{12}^{+}(2), \mathrm{P} \Omega_{12}^{-}(2) .
$$

Then all faithful primitive actions of $H$ of degree less than 4096 are on the cosets of reducible subgroups.

Proof. Throughout let $X$ be a proper irreducible subgroup of $H$ of largest possible order. For each $H$ we find $X$ or an upper bound on the order of $X$ and hence show that $|H: X|>4095$.

Linear. $H=\mathrm{L}_{n}(q)$ with $(n, q) \in\{(6,4),(6,5),(8,3)\}$. For each group $n$ is even and greater than 4 so $\operatorname{Soc}(X)=\mathrm{S}_{n}(q)$ by [20, Theorem 5.1]. This implies that $X=\mathrm{N}_{H}\left(\mathrm{~S}_{n}(q)\right) \leq \mathrm{N}_{\mathrm{PGL}_{n}(q)}\left(\mathrm{S}_{n}(q)\right)=\operatorname{PGSp}_{n}(q)$.

Symplectic. Let $H=\mathrm{S}_{6}(5)$ or $\mathrm{S}_{8}(3)$. Then by [20, Theorem 5.2], the group $X=\left(\mathrm{SL}_{2}(5) \curlywedge \mathrm{S}_{3}\right) / 2$ or $\mathrm{S}_{4}(9) .2$, respectively.

Unitary. For $H=\mathrm{U}_{5}(3)$ the group $X=\mathrm{N}_{H}\left(\mathrm{P} \Omega_{5}(3)\right)=\mathrm{PSO}_{5}(3)$ by [20, Theorem 5.3]. For $H=\mathrm{U}_{7}(2) \cong \mathrm{SU}_{7}(2)$ either $X=\left(\mathrm{GU}_{1}(2)\right.$ 乙 $\left.\mathrm{S}_{7}\right) \cap \mathrm{SU}_{7}(2)$ or $|X|<2^{18}$ by $[20$, Theorem 5.3, (iii)]. 
Orthogonal, odd dimension. Let $H=\mathrm{P} \Omega_{9}(3) \cong \Omega_{9}(3)$. By [20, Theorem $5.6]$ and divisibility consideridations one of the following holds

1. $X=\left(\mathrm{GO}_{3}(3)\right.$ 乙 $\left.\mathrm{S}_{3}\right) \cap \Omega_{9}(3)$;

2. $\operatorname{Soc}(X)=\mathrm{A}_{10}$;

3. $|X|<3^{22}$.

For case (2) let $Y:=\operatorname{Soc}(X)=\mathrm{A}_{10}$. Then $\mathrm{C}_{X}(Y) \unlhd X$, so if this centralizer were non-trivial, there would be some minimal normal subgroup $N$ of $X$ contained in $\mathrm{C}_{X}(Y)$. Then $N \leq \operatorname{Soc}(X) \cap \mathrm{C}_{X}(Y)=\mathrm{Z}(Y)=1$, which is a contradiction. Hence $\mathrm{C}_{X}(Y)=1$, so $X$ embeds in $\operatorname{Aut}(Y)$, namely $\mathrm{S}_{10}$.

Orthogonal, even dimension. By $\left[20\right.$, Theorems 5.4, 5.5] if $\operatorname{Soc}(H)=\mathrm{P} \Omega_{12}^{+}(2)$ $\cong \Omega_{12}^{+}(2)$ then $X=\mathrm{GU}_{6}(2) .2$ and if $\operatorname{Soc}(H)=\mathrm{P} \Omega_{12}^{-}(2) \cong \Omega_{12}^{-}(2)$ then $X \leq \mathrm{S}_{13}$.

For each of the above cases the index of $X$ in $H$ is greater than 4095 .

Let $H$ be one of the simple groups listed in Proposition 9. For each almost simple group with socle $H$ the results of $[12,13]$ are used to construct generators of conjugacy class representatives of reducible subgroups. If the index of a potentially maximal subgroup lies in the range $2500 \leq d<4096$ then we use MAGMA to check whether the corresponding permutation representation is primitive and keep those that are. This is described in detail below.

Linear. Let $H=\mathrm{L}_{n}(q)$ with $(n, q) \in\{(6,4),(6,5),(8,3)\}$. We construct the stabilizers of $k$-spaces for $1 \leq k \leq n / 2$ and the novelty reducible maximals of the extension of $H$ by the duality automorphism for $1 \leq k<n / 2$. The formulae for the corresponding group orders are found in [16, Propositions 4.1.17, 4.1.4, 4.1.22]. The only reducible subgroups of appropriate index are the stabilizers in $\mathrm{L}_{6}(5)$ and $\mathrm{L}_{8}(3)$ of a 1-dimensional subspace of index 3906 and 3280 respectively, both of which are maximal.

Symplectic. Let $H=\mathrm{S}_{n}(q)$ with $(n, q) \in\{(6,5),(8,3)\}$. We construct the stabilizers of a totally singular $k$-space for $1 \leq k \leq n / 2$ and a non-degenerate $k$-space for $2 \leq k<n / 2$ with $k$ even. The group orders are calculated using [16, Propositions 4.1.19, 4.1.3]. The 1-dimensional subspace stabilizers in $\mathrm{S}_{6}(5)$ and in $\mathrm{S}_{8}(3)$ have index 3906 and 3280, respectively, and both of these are maximal. All other stabilizers have index greater than 4095.

Unitary. Let $H=\mathrm{U}_{n}(q)$ with $(n, q) \in\{(5,3),(7,2)\}$. We construct the stabilizers of isotropic $k$-spaces for $1 \leq k \leq n / 2$ and of non-degenerate spaces for $1 \leq k<n / 2$. By [16, Propositions 4.1.18, 4.1.4] the only groups of appropriate index are the stabilizers in $\mathrm{U}_{7}(2)$ of an isotropic 1-space and a non-isotropic 1-space of index 2709 and 2752, respectively, both of which are maximal. 
Orthogonal, odd dimension. Let $H=\mathrm{P} \Omega_{9}(3)$. We find the stabilizers of totally singular $k$-spaces for $1 \leq k \leq 4$ and of non-degenerate $k$-spaces of plus and minus type for $1 \leq k<9$ and $k$ odd. The group orders are found in [16, Propositions 4.1.20, 4.1.6]. The groups of appropriate index are the stabilizers of a totally singular 1-space and a non-degenerate 8-space of plus or minus type of index 3280, 3321 and 3240, respectively, and all of these are maximal.

Orthogonal, even dimension. Let $H=\mathrm{P} \Omega_{12}^{+}(2)$ or $\mathrm{P} \Omega_{12}^{-}(2)$. We find the stabilizers of the following subspaces: A totally singular $k$-space for $1 \leq k \leq r$, where $r=6$ or 5 , respectively; a non-degenerate $k$-space of odd dimension, plus type or minus type for $1 \leq k \leq s$, where $s=5$ or 6 , respectively; and a non-singular 1-space. By [16, Propositions 4.1.20, 4.1.6, 4.1.7] none of these has index in the required range.

We conclude:

Theorem 10. Let $G$ be a primitive group of degree $2500 \leq d<4096$, with socle $H$ one of the groups in Proposition 9. Then $G$ appears in Tables 4 or 5.

\subsubsection{Actions on irreducible subgroups}

We classify the primitive permutation representations of degree $2500 \leq d<$ 4096 of $\mathrm{S}_{6}(4), \mathrm{S}_{12}(2), \mathrm{P} \Omega_{7}(5)$ and $\mathrm{L}_{12}(2)$ using Aschbacher's theorem [1]. The group $\mathrm{P} \Omega_{8}^{+}(3)$ is analysed separately [17]. The AS-maximals for classes $\mathcal{C}_{1}$ to $\mathcal{C}_{8}$ are given in [16, Section 4] and we find all potential maximals for subgroups in $\mathcal{C}_{9}$ using [22] and [11], which together list all absolutely irreducible representations of simple groups of small dimension.

Lemma 11. Let $G:=\mathrm{S}_{6}(4)$. If $M$ is a maximal subgroup of an almost simple group with socle $G$ of index less than 4096 then $M$ has index less than 2500.

Proof. The stabilizers of a totally singular $k$-space for $k \in\{1,2,3\}$ have index 1365,23205 and 5525 respectively. The stabilizer of a non-degenerate 2 -space has index 69888. The groups in $\mathcal{C}_{2}$ and $\mathcal{C}_{3}$ have index greater than 4095 , and Aschbacher classes $\mathcal{C}_{4}, \mathcal{C}_{6}$ and $\mathcal{C}_{7}$ are empty for groups with socle $\mathrm{S}_{6}(4)$. In $\mathcal{C}_{5}$ the stabilizer of a subfield of $\mathbb{F}_{4}$ of index 2 has index $\approx 3 \times 10^{6}$. The $\mathcal{C}_{8}$ groups $\mathrm{PGO}_{6}^{ \pm}(4)$ are maximal and have index 2080 and 2016 respectively. Potential maximal subgroups in $\mathcal{C}_{9}$ that are not groups of Lie type in defining characteristic have socle $\mathrm{U}_{3}(3), 2 . J_{2}, 2 . \mathrm{L}_{2}(13), 2 . \mathrm{L}_{2}(5)$ and $2 . \mathrm{L}_{2}(7)$, by [11]. For each group $M$ in this list $\left|\mathrm{S}_{6}(4)\right| /|\operatorname{Aut}(M)|>4095$. Potential maximals which are groups of Lie type in defining characteristic are $\mathrm{L}_{4}(2), \mathrm{L}_{4}(4), \mathrm{U}_{4}(2), \mathrm{U}_{4}(4)$ and $\mathrm{G}_{2}(4)$ by [22]. However $\mathrm{U}_{4}(4) \cong \mathrm{P} \Omega_{6}^{-}(4)$ and $\mathrm{L}_{4}(4) \cong \mathrm{P} \Omega_{6}^{+}(4)$, which are both in $\mathcal{C}_{8}$, and if $M$ is one of $\mathrm{L}_{4}(2), \mathrm{U}_{4}(2)$, or $\mathrm{G}_{2}(4)$ then $\left|\mathrm{S}_{6}(4)\right| /|\operatorname{Aut}(M)|>4095$.

Lemma 12. Let $G:=\mathrm{S}_{12}(2) \cong \operatorname{Aut}\left(\mathrm{S}_{12}(2)\right)$. If $M$ is a maximal subgroup of $G$ of index less than 4096 and at least 2500 then $M$ is the stabilizer of a totally isotropic 1-space of index 4095; 
Proof. The stabilizer of a 1-space has index 4095 whilst all other totally singular $k$-space stabilizers have index greater than 4095 . The stabilizers of a non-degenerate $k$-space for $k \in\{2,4\}$ both have index greater than 4095. The AS-maximals in $\mathcal{C}_{2}$ all have index greater than 4095. There are two AS-maximal groups in $\mathcal{C}_{3}$, namely $\mathrm{S}_{6}(4) .2$ and $\mathrm{S}_{4}(8) .3$, both of which have index greater than 4095. There are no groups in Aschbacher classes $\mathcal{C}_{i}$ with $4 \leq i \leq 7$ for groups with socle $\mathrm{S}_{12}(2)$. In class $\mathcal{C}_{8}$ the AS-maximals are $\mathrm{PGO}_{12}^{+}(2)$ of index 2080 and $\mathrm{PGO}_{12}^{-}(2)$ of index 2016. Let $G$ be a $\mathcal{C}_{9}$ maximal. Then one of $\operatorname{Soc}(G) \cong \mathrm{A}_{13}$, $\operatorname{Soc}(G) \cong \mathrm{A}_{14}$ or $|G|<2^{36}$ holds by [20, Theorem 4.1]. Hence the smallest possible index of $G$ is $\left|\mathrm{S}_{12}(2)\right| / 14$ ! > 4095 .

Lemma 13. If $M$ is a maximal subgroup of an almost simple group $G$ with socle $\mathrm{P} \Omega_{7}(5)$ and $M$ has index less than 4096 , then $M$ stabilizes a totally singular 1space, $|G: M|=3906$ and $\mathrm{N}_{S}\left(M \cap \mathrm{P} \Omega_{7}(5)\right)$ is maximal in all almost simple groups $S$ with socle $\mathrm{P} \Omega_{7}(5)$.

Proof. The stabilizer of a totally singular $k$-space has index 3906 when $k=1$ and when $k \geq 2$ the index is greater than 4095 . For $1 \leq k \leq 5$ the stabilizer of a non-degenerate $k$-space (with $k$ odd) has index $>4095$. The only ASmaximal group in $\mathcal{C}_{2}$ has shape $2^{6} . A_{7}$ and index $\approx 10^{9}$. There are no groups in Aschbacher classes $\mathcal{C}_{i}$ with $3 \leq i \leq 8$ for groups with socle $\mathrm{P} \Omega_{7}(5)$. Potential $\mathcal{C}_{9}$ maximals that are not groups of Lie type in defining characteristic have socle $\mathrm{A}_{8}, \mathrm{~S}_{6}(2), \mathrm{L}_{2}(7), \mathrm{L}_{2}(8)$ and $\mathrm{U}_{3}(3)$ by [11]. For each group $M$ in this list $\left|\mathrm{P} \Omega_{7}(5)\right| /|\operatorname{Aut}(M)|>4095$. The only potential maximal subgroup of Lie type in defining characteristic is $\mathrm{G}_{2}(5) \cong \operatorname{Aut}\left(\mathrm{G}_{2}(5)\right)$ by [22], which has index greater than 4095 .

Lemma 14. Let $G$ be an almost simple group with socle $\mathrm{L}_{12}(2)$. If $M$ is a maximal subgroup of $G$ of index $2500 \leq d<4096$ then $M$ is the stabilizer in $G$ of a totally isotropic 1-space and has index 4095.

Proof. The stabilizer in $G$ of a totally singular $k$-space for $2 \leq k \leq 10$ has index greater than 4095. The stabilizers in $G$ of a totally isotropic 1-space and a totally isotropic 11-space have index 4095 and are conjugate in $\operatorname{Aut}\left(\mathrm{L}_{12}(2)\right)$. Thus the novelty $\mathcal{C}_{1}$ maximals all have index greater than 4095. Aschbacher classes $\mathcal{C}_{i}$, with $5 \leq i \leq 7$ are empty for groups with socle $\mathrm{L}_{12}(2)$ and all subgroups in classes $\mathcal{C}_{2}, \mathcal{C}_{3}, \mathcal{C}_{4}$ and $\mathcal{C}_{8}$ have index greater than 4095 .

From [11] and [22] the possible $\mathcal{C}_{9}$ maximals of $G$ have socle $6 . \mathrm{A}_{6}, 3 . \mathrm{Suz}$, $\mathrm{S}_{12}(2)$ and $\mathrm{P} \Omega_{12}^{ \pm}(2)$. The groups $\mathrm{S}_{12}(2)$ and $\mathrm{P} \Omega_{12}^{ \pm}(2)$ are in $\mathcal{C}_{8}$ and the representations of $6 . \mathrm{A}_{6}$ and 3. Suz need a cube root of unity, hence are not in $\mathrm{L}_{12}(2)$.

Lemma 15. If $G$ is a group with socle $\mathrm{P} \Omega_{8}^{+}(3)$ then $G$ has no primitive permutation representation of degree less than 4096.

Proof. The list of maximal subgroups of the almost simple groups with socle $\mathrm{P} \Omega_{8}^{+}(3)$ in [6] is not claimed to be complete. We used [17] to verify that no maximal subgroup has index $2500 \leq d<4096$. 
We conclude:

Theorem 16. The primitive almost simple classical groups of degree $2500 \leq$ $d<4096$ are given in Tables 4 and 5 .

\subsection{Exceptional and sporadic groups}

We use Lemma 5 and consider only the simple exceptional groups. Let $G:=\operatorname{Ch}(q)$ be a Chevalley group with $q=p^{e}$ and define $l(G, p)>1$ to be the smallest possible degree of a non-trivial projective irreducible representation of $G$ over a field of characteristic other than $p$. A primitive permutation representation of degree $d$ corresponds to a (not necessarily irreducible) representation by permutation matrices in coprime characteristic in dimension $d-1$. Hence a lower bound for $l(G, p)$ gives a lower bound for $P(G)$.

Proposition 17. Let $G$ be an almost simple exceptional group with a faithful primitive permutation representation of degree $2500 \leq d<4096$. Then $\operatorname{Soc}(G)$ is one of $\mathrm{G}_{2}(3), \mathrm{G}_{2}(5)$ or ${ }^{2} \mathrm{~F}_{4}(2)^{\prime}$.

Proof. To begin with we examine the untwisted groups: $\mathrm{E}_{6}(q), \mathrm{E}_{7}(q), \mathrm{E}_{8}(q)$, $\mathrm{F}_{4}(q)$ and $\mathrm{G}_{2}(q)$. Secondly we deal with the twisted groups: ${ }^{2} \mathrm{~B}_{2}\left(2^{2 m+1}\right)=$ $\mathrm{Sz}\left(2^{2 m+1}\right),{ }^{3} \mathrm{D}_{4}(q),{ }^{2} \mathrm{E}_{6}(q),{ }^{2} \mathrm{~F}_{4}(q)$ and ${ }^{2} \mathrm{G}_{2}\left(3^{2 m+1}\right)$.

The minimal degree $P\left(\mathrm{E}_{6}(q)\right) \geq q^{9}\left(q^{2}-1\right)$ by [19], so it suffices to consider $q=2$. However all maximal subgroups of $\mathrm{E}_{6}(2)$ have index greater than 4095 by [18]. The values of $l\left(\mathrm{E}_{7}(q), p\right)=q^{15}\left(q^{2}-1\right)$ and $l\left(\mathrm{E}_{8}(q), p\right)=q^{27}\left(q^{2}-1\right)$ are greater than 4095 for all $q$.

The smallest degree $l\left(\mathrm{~F}_{4}(q), p\right) \geq q^{9} / 2>4095$ for all $q>2$ by [19], and the index of the largest maximal subgroup of $\mathrm{F}_{4}(2)$ is 69615 from [6].

The largest maximal subgroup of $\mathrm{G}_{2}(q)$ has index greater than $q^{5}+q^{4}+$ $q^{3}+q^{2}+q+1$ for $q>4$ by [21, Theorem 5.2] and therefore $P\left(\mathrm{G}_{2}(q)\right)>4095$ for all $q>5$. The group $\mathrm{G}_{2}(2)$ is not simple and $\mathrm{G}_{2}(2)^{\prime}$ is isomorphic to $\mathrm{U}_{3}(3)$. The group $\mathrm{G}_{2}(3)$ has three maximal subgroups of index $2500 \leq d<4096$ and $\operatorname{Aut}\left(\mathrm{G}_{2}(3)\right)$ has no novelties with index in that range [6]. The group $\mathrm{G}_{2}(4)$ has no maximal subgroups of index $2500 \leq d<4096$, nor does $\operatorname{Aut}\left(\mathrm{G}_{2}(4)\right) \cong \mathrm{G}_{2}(4) .2$ by [6]. The group $\mathrm{G}_{2}(5) \cong \operatorname{Aut}\left(\mathrm{G}_{2}(5)\right)$ has two maximal subgroups of index 3906 by $[6]$.

Now we analyse the twisted groups. The smallest degree $P\left(\operatorname{Sz}\left(2^{2 m+1}\right)\right)>$ 4095 for $m \geq 4$ by [19]. No maximal subgroups of $\mathrm{Sz}(8)$ and $\mathrm{Sz}(32)$ or their automorphism groups have index in the range $2500 \leq d<4096$ by [6]. Let $G:=\mathrm{Sz}(q)$ and let $r^{2}=2 q$, so that $|G|=q^{2}(q-1)\left(q^{2}+1\right)$. Then the possible orders of a maximal subgroup of $G$ are $\left\{q^{2}(q-1), 2(q-1), 4(q+r+1), 4(q-\right.$ $\left.r+1),\left|\mathrm{Sz}\left(q_{0}\right)\right|: q_{0}^{t}=q\right\}$ by [36, p137, Theorem 9]. Hence for $m \geq 3$ the group $\mathrm{Sz}\left(2^{2 m+1}\right)$ has no proper subgroups of index less than 4095 .

A maximal subgroup of ${ }^{3} \mathrm{D}_{4}(q)$ has index at least $q^{8}+q^{4}+1$ by $[21$, Theorem 5.2 ], so for $q>2$ all maximal subgroups of ${ }^{3} \mathrm{D}_{4}(q)$ have index larger than 4095 . No almost simple group with socle ${ }^{3} \mathrm{D}_{4}(2)$ has a maximal subgroup of index $2500 \leq d<4096$ by [6]. 
The minimal degree $l\left({ }^{2} \mathrm{E}_{6}(q), p\right) \geq q^{8}\left(q^{4}+1\right)\left(q^{3}-1\right)$ by [19], which is greater than 4095 for $q>2$. No almost simple group with socle ${ }^{2} \mathrm{E}_{6}(2)$ has a maximal subgroup of index less than 4096 by [6].

A maximal subgroup of ${ }^{2} \mathrm{~F}_{4}(q)$ with $q>2$ has index at least $\left(q^{6}+1\right)\left(q^{3}+\right.$ $1)(q+1)$ by [21, Theorem 5.2] and so $P\left({ }^{2} \mathrm{~F}_{4}(q)\right)>4095$ for $q>2$. The groups ${ }^{2} \mathrm{~F}_{4}(2)^{\prime}$ and Aut $\left({ }^{2} \mathrm{~F}_{4}(2)^{\prime}\right)$ each have a maximal subgroup of index 2925 by [6].

Lastly, ${ }^{2} \mathrm{G}_{2}(3) \cong \mathrm{L}_{2}(8): 3$ and $l\left({ }^{2} \mathrm{G}_{2}\left(3^{2 m+1}\right), p\right) \geq 3^{2 m+1}\left(3^{2 m+1}-1\right)$ for $m>$ 0 . If $m \geq 2$ then $P\left({ }^{2} \mathrm{G}_{2}\left(3^{2 m+1}\right)\right)>4095$ which leaves only ${ }^{2} \mathrm{G}_{2}\left(3^{3}\right) \cong \mathrm{R}(27)$ which has no maximal subgroups of index less than 4096 by [6].

Information about the maximal subgroups of the 26 sporadic groups is in [6] and corrected in [31]. With the exception of the Monster group M, the list of maximal subgroups is complete for each group. The smallest dimension of an irreducible complex representation of $\mathrm{M}$ is 196883 , hence $\mathrm{M}$ has no transitive permutation of degree $<4096$. The sporadic groups with primitive permutation representations of degree $2500 \leq d<4096$ are $\mathrm{J}_{1}, \mathrm{HS}, \mathrm{M}_{24}, \mathrm{Ru}$ and $\mathrm{Fi}_{22}$. All of the exceptional groups are also in [6]. We conclude:

Theorem 18. The exceptional and sporadic groups with faithful primitive permutation representations of degree $2500 \leq d<4096$ are given in Table 6 .

\section{Product action groups}

We classify the primitive product action groups of degree $2500 \leq d<4096$.

Definition 19. Let $B$ be a group acting on a set $\Delta$ and let $W:=B \imath \mathrm{S}_{k}$. The product action of $\left(b_{1}, \ldots, b_{k}\right) \sigma \in W$ on $\left(\delta_{1}, \ldots, \delta_{k}\right) \in \Delta^{k}$ is defined as follows.

$$
\left(\delta_{1}, \ldots, \delta_{k}\right)^{\left(b_{1}, \ldots, b_{k}\right) \sigma}=\left(\delta_{1}^{b_{1}}, \ldots, \delta_{k}^{b_{k}}\right)^{\sigma}=\left(\delta_{1 \sigma^{-1}}^{b_{1 \sigma^{-1}}}, \ldots, \delta_{k \sigma^{-1}}^{b_{k \sigma-1}}\right) .
$$

Let $P$ be a primitive group of almost simple or diagonal type of degree $n$. Then $K:=\operatorname{Soc}(P)$ is isomorphic to a direct power $T^{l}=T \times \cdots \times T$ of a nonabelian simple group $T$. Let $m \geq 2$ be a non-trivial multiple of $l$ and let $W$ be the product action wreath product $P \prec \mathrm{S}_{m / l}$. We list the groups $G$ such that

$$
K^{m / l} \leq G \leq W
$$

and $G$ is a primitive permutation group of degree $d=n^{m / l}$.

Since $P$ is of almost simple or diagonal type, $n \geq 5$. The condition $2500 \leq$ $d<4096$ implies $m \leq 5$ and the following values can occur:

- $m / l=2$ and $50 \leq n<64$

- $m / l=3$ and $14 \leq n<16$

- $m / l=5$ and $n=5$. 
The primitive groups of degree less than 64 are in the primitive groups library of Magma. Let $P$ be the largest primitive group in its cohort. The only group of diagonal type in this list is $P=\mathrm{A}_{5}^{2} \cdot 2^{2}$, the socle of which is isomorphic to $\mathrm{A}_{5} \times \mathrm{A}_{5}$. In this case $l=2$ and $m=4$, for all other groups $l=1$.

To find the primitive groups of product action type we proceed as follows. For each $P$ construct the product action wreath product $W:=P \prec \mathrm{S}_{m / l}$ and take the socle quotient $W / \operatorname{Soc}(W)$. The preimages of the primitive subgroups of this quotient are primitive groups of degree $n^{m / l}$.

Theorem 20. The product action type primitive permutation groups of degree $2500 \leq d<4096$ are given in Table 8 .

\section{Diagonal type groups}

Lastly we consider the diagonal type groups. Let $T$ be a non-abelian simple group, let $m \geq 2$ and consider a group $W$ of shape $\left(T 2 \mathrm{~S}_{m}\right)$. Out $(T)$. The diagonal subgroup of $W$ is $D=\{(t, t, \ldots, t) \mid t \in T\}$. $\left(\mathrm{S}_{m} \times \operatorname{Out}(T)\right)$ and the action of $W$ on the cosets of $D$ is the diagonal action. A permutation group $G$ is of diagonal type if $T^{m} \leq G \leq W$ with the diagonal action. Then $\operatorname{Soc}(G) \cong T^{m}$, the degree of $G$ is $d:=|T|^{m-1}$ and the full normalizer $N$ of $T^{m}$ in $\mathrm{S}_{d}$ is equal to $W$.

Theorem 21. [8, p123] A diagonal type group $G \leq \mathrm{S}_{d}$ is primitive if and only if either $m=2$ or $m \geq 3$ and the conjugation action of $G$ on the set of all minimal normal subgroups of $T^{m}$ is primitive.

Since we are only interested in the primitive groups of degree $2500 \leq d=$ $|T|^{m-1}<4096$ the possible simple groups $T$ are $\mathrm{A}_{7}, \mathrm{~L}_{2}(19), \mathrm{L}_{2}(16)$ with $m=2$ and $\mathrm{A}_{5}$ with $m=3$.

\subsection{Method}

Let $W:=\operatorname{Aut}(T) \prec \mathrm{S}_{m}$. Then $W$ has subgroups isomorphic to all groups of diagonal type with socle $T^{m}$. A group isomorphic to $N_{\mathrm{S}_{d}}\left(T^{m}\right)$ lies in the set

$$
\Lambda=\left\{K \leq W:|W: K|=|\operatorname{Out}(T)|^{m-1}\right\} .
$$

Using MAgma to compute $\Lambda$ for each $T$ given above we see there is only one group $L$ which has a maximal subgroup of index $|T|^{m-1}$ in each case. For each such maximal subgroup $M$ of $L$ we let $N$ be the permutation representation of $L$ acting on the cosets of $M$. When $m=2$ the primitive groups are found by taking all subgroups of the socle quotient $N / \operatorname{Soc}(N)$ and storing their preimages.

When $m=3$ the action of $G$ by conjugation on the set of all minimal normal subgroups of $T^{3}$ is primitive. In this case $T=\mathrm{A}_{5}$ and

$$
N:=\left(\mathrm{A}_{5}\right)^{3}:\left(\mathrm{S}_{3} \times 2\right)
$$

so in a primitive subgroup $G \leq N$ the minimal normal subgroups of the socle are permuted by a subgroup of $S_{3}$. The only primitive subgroups of $S_{3}$ are itself and $\mathrm{A}_{3}$, hence the primitive subgroups of $N$ are those with either $\mathrm{S}_{3}$ or $\mathrm{A}_{3}$ in their socle quotients. This enables us to prove the following theorem. 
Theorem 22. The primitive permutation groups of diagonal type of degree $2500 \leq d<4096$ are given in Table $\%$.

This completes our classification of primitive groups.

\section{Accuracy checks}

This section details the methods used to check our results. We also state which results in the literature have been used without rechecking.

Where there are families of groups in [32] (such as $\left\{\mathrm{A}_{n}: 5 \leq n \leq 71\right\}$ ) which can be extended to include groups of degree $2500 \leq d<4096$ the parameters of the smallest group of the new family were compared to those of the largest member of the existing family to ensure there are no omissions.

The soluble irreducible subgroups of $\mathrm{GL}_{k}(p)$ for $p^{k}<2^{16}$ are in the IRREDSOL package of GAP and these were checked against MAGMA, finding no discrepancy. Our numbers of soluble groups agree with those in [9].

We have made extensive use of [16] and in particular have assumed the results of Section 4 to be accurate. The primitive permutation groups of degree less than 2500 given in [32] are assumed to be correct without rechecking. The other main references whose accuracy has been relied upon are [20, Theorems 5.1-5.5] for bounds on the orders of maximal irreducible subgroups of classical groups, [11] and [22] for the $\mathcal{C}_{9}$ maximals of almost simple groups, [17] for the maxmal subgroups of $\mathrm{P} \Omega_{8}^{+}(3)$, [19] for bounds on degrees of permutation representations of the exceptional groups and [21, Theorom 5.2] for bounds on the orders of maximal subgroups of some exceptional groups. We frequently used [6] and each time consulted [31] to ensure accuracy.

The definition of product action primitive groups given in Section 5 is more restrictive than that in [8] to make the O'Nan-Scott classes disjoint.

Lemma 23. A primitive group $G$ belongs to exactly one O'Nan-Scott class.

Proof. The socle of $G$ is abelian if and only if $G$ is of affine type. The socle of $G$ is nonabelian and regular if and only if $G$ is a twisted wreath product. The socle of $G$ is non-abelian simple if and only if $G$ is an almost simple group.

Thus we assume that $\operatorname{Soc}(G) \cong T^{m}$ for some non-abelian simple group $T$ and $m \geq 2$, with nontrivial point stabilisers. We need only show that if $H \cong G$ and $H$ is primitive of the same degree as $G$ then $H$ and $G$ are not of product action and diagonal type, respectively. Assume otherwise, then $T^{m} \leq G \leq$ $T^{m}$. $\left(\mathrm{S}_{m} \times \operatorname{Out}(T)\right)$ and $T^{m} \cong S^{k} \leq H \leq P^{k}: \mathrm{S}_{k}$, where $P$ is a primitive group of almost simple or diagonal type, $k>1$ divides $m$ and $\operatorname{Soc}(P)=S \cong T^{m / k}$. If $P$ is almost simple then $k=m$ and $H_{\alpha}$ does not contain $T$. This is not the case for $G$, so $P$ is of diagonal type. Then $S \cong T^{l}$ for some $l=m / k>1$, and $P$ has degree $|T|^{l-1}$. Now $\operatorname{Soc}(G) \cong T^{k l}$ and $G$ has degree $|T|^{k l-1}$, however the degree of $H$ is $\left(|T|^{l-1}\right)^{k}=|T|^{k l-k}$, so $G$ is not isomorphic to $H$.

Hence we only check for permutation isomorphism between groups of the same degree within the O'Nan-Scott classes and this was done using the same 


\begin{tabular}{cll} 
Table 2: & \multicolumn{2}{c}{ Primitive groups of affine type } \\
\hline$p^{k}$ & Soluble & Insoluble \\
\hline $53^{2}$ & 100 & 6 \\
$59^{2}$ & 82 & 6 \\
$61^{2}$ & 212 & 20 \\
$5^{5}$ & 48 & 46 \\
\hline
\end{tabular}

methods as [32]. The signature of a group $G$ is the following list of properties: the order of $G$, the largest integer $k$ such that $G$ acts $k$-transitively, the multiset of orbit lengths of the $k$-point stabilizer of $G$, the multiset of chief factors of $G$ and the orders of all groups in the derived series of $G$. Adding to the signature the multisets of isomorphism types of all abelian groups that both occur as quotients in the derived series of $G$ and are in the small groups library of MAGMA gives us the extended signature of $G$.

Let L be a list of groups of the same degree in a particular O'Nan-Scott class. We partition the groups in L using their signatures and delete from L any groups in a class of size 1 . Next we compute the Sylow 2-subgroup $S$ of each group in L and repeat the process now using the extended signature of $S$, again discarding the groups in equivalence classes of size 1 . Now the point stabilizer and derived subgroup of the remaining groups in L are computed and the groups are again partitioned by their extended signature. The number of groups remaining after this step is small enough for us to check by hand that no pair of groups is permutation isomorphic. This test was carried out for each collection of groups of the same degree inside an O'Nan-Scott class.

To avoid computational errors we have repeatedly run the code and checked that the results agree. When using [12] to construct the maximal reducible subgroups of a group we ensured maximality by checking that the groups arising from them are primitive. The groups declared to be CS and the primitive groups database have been in use in MAGMA for a number of years.

\section{Tables}

We now give tables of the primitive permutation groups of degree $2500 \leq$ $d<4096$. Recall that $q$ is always a prime power, $p$ is always a prime and $n$ is a positive integer. The dihedral group of order $2 n$ is denoted $\mathrm{D}_{2 n}$ and $[n]$ denotes a soluble group of order $[n]$.

The table for the affine groups lists the numbers of soluble and insoluble primitive subgroups of degree $p^{k}$ for $k>1$. The number of primitive subgroups of $\mathrm{AGL}_{1}(p) \cong p:(p-1)$ is equal to the number of divisors of $p-1$ and hence is omitted from our tables. For each of the other O'Nan-Scott classes the first column of each table contains the smallest group $G$ of the cohort. Also given are the degree $d$ of $G$, the rank (number of orbits of a point stabilizer) of the normalizer $N$ of $G$ in $\mathrm{S}_{d}$ and the number of groups in the cohort of $G$. In the 
Table 3: Primitive almost simple groups with alternating socle

\begin{tabular}{lllllll}
\hline $\begin{array}{l}\text { Primitive } \\
\text { group } G\end{array}$ & Conditions & Degree & $\begin{array}{l}\text { Stabilizer } \\
\text { in } G\end{array}$ & $N$ & $\begin{array}{l}\text { Rank } \\
\text { of } N\end{array}$ & $\begin{array}{l}\text { Cohort } \\
\text { size }\end{array}$ \\
\hline $\mathrm{A}_{n}$ & $72 \leq n \leq 91$ & $\left(\begin{array}{l}n \\
2\end{array}\right)$ & $\mathrm{S}_{n-2}$ & $H .2$ & 3 & 2 \\
Out $=2$ & $\begin{array}{l}26 \leq n \leq 30 \\
3 \\
3\end{array}$ & $\left(\mathrm{~A}_{n-3} \times 3\right): 2$ & $H .2$ & 4 & 2 \\
& $18 \leq n \leq 19$ & $\left(\begin{array}{l}n \\
4\end{array}\right)$ & $\left(\mathrm{A}_{n-4} \times \mathrm{A}_{4}\right): 2$ & $H .2$ & 5 & 2 \\
\hline $\mathrm{A}_{10}$ & & 2520 & $\mathrm{M}_{10}$ & $H .2$ & 10 & 2 \\
$\mathrm{~A}_{11}$ & & 2520 & $\mathrm{M}_{11}$ & $H$ & 5 & 1 \\
$\mathrm{~A}_{12}$ & & 2520 & $\mathrm{M}_{12}$ & $H$ & 4 & 1 \\
$\mathrm{~A}_{14}$ & & 3003 & $\left(\mathrm{~A}_{8} \times \mathrm{A}_{6}\right): 2$ & $H .2$ & 7 & 2 \\
$\mathrm{~A}_{15}$ & & 3003 & $\left(\mathrm{~A}_{10} \times \mathrm{A}_{5}\right): 2$ & $H .2$ & 6 & 2 \\
\hline
\end{tabular}

Table 4: Primitive almost simple groups with socle $\mathrm{L}_{2}(q)$

\begin{tabular}{lllllll}
\hline $\begin{array}{l}\text { Primitive } \\
\text { group } G\end{array}$ & Conditions & Degree & $\begin{array}{l}\text { Stabilizer } \\
\text { in } G\end{array}$ & $N$ & $\begin{array}{l}\text { Rank } \\
\text { of } N\end{array}$ & $\begin{array}{l}\text { Cohort } \\
\text { size }\end{array}$ \\
\hline $\mathrm{L}_{2}(p)$ & $73 \leq p \leq 89$ & $\left(\begin{array}{c}p \\
2\end{array}\right)$ & $\mathrm{D}_{p+1}$ & $H .2$ & $\frac{p+1}{2}$ & 2 \\
$\mathrm{~L}_{2}(p)$ & $71 \leq p \leq 89$ & $\left.\begin{array}{c}p+1 \\
2\end{array}\right)$ & $\mathrm{D}_{p-1}$ & $H .2$ & $\frac{p+3}{2}$ & 2 \\
$\mathrm{~L}_{2}(p)$ & $2503 \leq p \leq 4093$ & $p+1$ & $p:(p-1) / 2$ & $H .2$ & 2 & 2 \\
$\mathrm{~L}_{2}(43)$ & & 3311 & $\mathrm{~A}_{4}$ & $H .2$ & 152 & 2 \\
$\mathrm{~L}_{2}(71)$ & & 2982 & $\mathrm{~A}_{5}$ & $H$ & 61 & 1 \\
\hline $\mathrm{L}_{2}\left(p^{2}\right)$ & $53 \leq p \leq 61$ & $p^{2}+1$ & $p^{2}:\left(p^{2}-1\right) / 2$ & $H .2^{2}$ & 2 & 5 \\
$\mathrm{~L}_{2}\left(19^{2}\right)$ & & 3439 & $\mathrm{PGL}_{2}(19)$ & $H .2$ & 11 & 2 \\
$\mathrm{~L}_{2}\left(3^{4}\right)$ & & 3240 & $\mathrm{D}_{82}$ & $H .2^{3}$ & 14 & 8 \\
& & 3321 & $\mathrm{D}_{80}$ & $H .2^{3}$ & 15 & 8 \\
$\mathrm{~L}_{2}\left(5^{5}\right)$ & & $5^{5}+1$ & $5^{5}:\left(5^{5}-1\right) / 2$ & $H .5 .2$ & 2 & 4
\end{tabular}

tables of the almost simple groups we also give the structure of $N$ in terms of the socle $H$ of $G$, and the shape of a point stabilizer in $G$. Note that Table 3 does not contain $\mathrm{A}_{d}$ and $\mathrm{S}_{d}$ in their natural action. 
Table 5: Primitive almost simple groups with other classical socles

\begin{tabular}{|c|c|c|c|c|c|}
\hline $\begin{array}{l}\text { Primitive } \\
\text { group } G\end{array}$ & Degree & $\begin{array}{l}\text { Stabilizer } \\
\text { in } G\end{array}$ & $N$ & $\begin{array}{l}\text { Rank } \\
\text { of } N\end{array}$ & $\begin{array}{l}\text { Cohort } \\
\text { size }\end{array}$ \\
\hline \multirow[t]{3}{*}{$\mathrm{L}_{3}(5)$} & 3100 & $\mathrm{~S}_{5}$ & $H .2$ & 32 & 2 \\
\hline & 3875 & $4^{2}: \mathrm{S}_{3}$ & $H .2$ & 35 & 2 \\
\hline & 4000 & $31: 3$ & $H .2$ & 35 & 2 \\
\hline $\mathrm{L}_{3}(7) .2$ & 2793 & $2^{\cdot}\left(\mathrm{L}_{2}(7) \times 2\right) .2$ & $H . \mathrm{S}_{3}$ & 10 & 2 \\
\hline $\mathrm{L}_{3}(13) .2$ & 2562 & {$\left[13^{3}\right] \cdot[48] .2$} & $H . \mathrm{S}_{3}$ & 4 & 2 \\
\hline $\mathrm{L}_{3}(53)$ & 2863 & $53^{2} \cdot[52] . \mathrm{L}_{2}(53) .2$ & $H$ & 2 & 1 \\
\hline $\mathrm{L}_{3}(59)$ & 3541 & $59^{2} \cdot[58] \cdot \mathrm{L}_{2}(59) \cdot 2$ & $H$ & 2 & 1 \\
\hline $\mathrm{L}_{3}(61)$ & 3783 & $61^{2} \cdot[20] \cdot \mathrm{L}_{2}(61) .2$ & $H .3$ & 2 & 2 \\
\hline $\mathrm{L}_{4}(7)$ & 2850 & {$\left[7^{4}\right] \cdot[6] \cdot \mathrm{L}_{2}(7)^{2} .2$} & $H .2^{2}$ & 3 & 5 \\
\hline $\mathrm{L}_{5}(7)$ & 2801 & {$\left[7^{4}\right] .[6] . \mathrm{L}_{4}(7) .2$} & $H$ & 2 & 1 \\
\hline $\mathrm{L}_{6}(5)$ & 3906 & {$\left[5^{5}\right] .2 . \mathrm{L}_{5}(5)$} & $H .2$ & 2 & 2 \\
\hline $\mathrm{L}_{7}(2)$ & 2667 & {$\left[2^{10}\right] \cdot\left(\mathrm{S}_{3} \times \mathrm{L}_{5}(2)\right)$} & $H$ & 3 & 1 \\
\hline $\mathrm{L}_{8}(3)$ & 3280 & {$\left[3^{7}\right] \cdot \mathrm{L}_{7}(3)$} & $H .2$ & 2 & 2 \\
\hline $\mathrm{L}_{12}(2)$ & 4095 & {$\left[2^{11}\right] \cdot \mathrm{L}_{11}(2)$} & $H$ & 2 & 1 \\
\hline \multirow[t]{2}{*}{$\mathrm{S}_{4}\left(3^{2}\right)$} & 3240 & $\mathrm{~S}_{2}\left(3^{4}\right) .2$ & $H .2^{2}$ & 5 & 5 \\
\hline & 3321 & $2 . \mathrm{S}_{2}\left(3^{2}\right) \succ 2$ & $H .2^{2}$ & 5 & 5 \\
\hline $\mathrm{S}_{6}(3)$ & 3640 & $3^{3+4}: 2 .\left(\mathrm{S}_{4} \times \mathrm{A}_{4}\right)$ & $H .2$ & 5 & 2 \\
\hline $\mathrm{S}_{6}(5)$ & 3906 & {$\left[5^{5}\right]: 4 . \mathrm{S}_{4}(5)$} & $H .2$ & 3 & 2 \\
\hline $\mathrm{S}_{8}(3)$ & 3280 & {$\left[3^{7}\right]: 2 . \mathrm{S}_{6}(3)$} & $H .2$ & 3 & 2 \\
\hline $\mathrm{S}_{12}(2)$ & 4095 & {$\left[2^{11}\right]: \mathrm{S}_{10}(2)$} & $H$ & 3 & 1 \\
\hline $\mathrm{U}_{3}\left(2^{3}\right)$ & 3648 & $3 \times \mathrm{L}_{2}(8)$ & $H .\left(3 \times \mathrm{S}_{3}\right)$ & 5 & 9 \\
\hline $\mathrm{U}_{4}(3)$ & 2835 & 2. $\left(\mathrm{A}_{4} \times \mathrm{A}_{4}\right) \cdot 2^{2}$ & $H . \mathrm{D}_{8}$ & 9 & 8 \\
\hline $\mathrm{U}_{4}\left(2^{2}\right)$ & 3264 & $5 . \mathrm{U}_{3}(4)$ & $H .4$ & 4 & 3 \\
\hline $\mathrm{U}_{4}(5)$ & 3276 & {$\left[5^{5}\right]:[12] \cdot \mathrm{U}_{2}(5) .2$} & $H .2^{2}$ & 3 & 5 \\
\hline $\mathrm{U}_{4}(7)$ & 2752 & {$\left[7^{4}\right]: 3 \cdot \mathrm{L}_{2}\left(7^{2}\right)$} & $H . \mathrm{D}_{8}$ & 3 & 8 \\
\hline $\mathrm{U}_{5}(2)$ & 3520 & $\mathrm{~S}_{3} \times 3^{1+2}: 2 \mathrm{~A}_{4}$ & $H .2$ & 12 & 2 \\
\hline \multirow[t]{2}{*}{$\mathrm{U}_{7}(2)$} & 2709 & {$\left[2^{11}\right]: 3 . \mathrm{U}_{5}(2)$} & $H .2$ & 3 & 2 \\
\hline & 2752 & $3 . \mathrm{U}_{6}(2) .3$ & $H .2$ & 3 & 2 \\
\hline \multirow[t]{2}{*}{$P \Omega_{7}(3)$} & 3159 & $\mathrm{~S}_{6}(2)$ & $H$ & 4 & 1 \\
\hline & 3640 & $3^{1+6}:\left(2 \mathrm{~A}_{4} \times \mathrm{A}_{4}\right) \cdot 2$ & $H .2$ & 5 & 2 \\
\hline $\mathrm{P} \Omega_{7}(5)$ & 3906 & {$\left[5^{5}\right]:\left(2 \times \Omega_{5}(5)\right) .2$} & $H .2$ & 3 & 2 \\
\hline \multirow{3}{*}{$P \Omega_{9}(3)$} & 3240 & $\Omega_{8}^{-}(3) .2$ & $H .2$ & 3 & 2 \\
\hline & 3280 & {$\left[3^{7}\right]: \Omega_{7}(3) .2$} & $H .2$ & 3 & 2 \\
\hline & 3321 & $\Omega_{8}^{+}(3) .2$ & $H .2$ & 3 & 2 \\
\hline
\end{tabular}


Table 6: Primitive almost simple groups with exceptional and sporadic socle

\begin{tabular}{llllll}
\hline $\begin{array}{l}\text { Primitive } \\
\text { group } G\end{array}$ & Degree & $\begin{array}{l}\text { Stabilizer } \\
\text { in } G\end{array}$ & $N$ & $\begin{array}{l}\text { Rank } \\
\text { of } N\end{array}$ & $\begin{array}{l}\text { Cohort } \\
\text { size }\end{array}$ \\
\hline $\mathrm{G}_{2}(3)$ & 2808 & $\mathrm{~L}_{2}(8): 3$ & $H: 2$ & 7 & 2 \\
& 3159 & $2^{3} \cdot \mathrm{L}_{3}(2)$ & $H: 2$ & 8 & 2 \\
& 3888 & $\mathrm{~L}_{2}(13)$ & $H: 2$ & 12 & 2 \\
${ }^{2} \mathrm{~F}_{4}(2)^{\prime}$ & 2925 & $2^{2} \cdot\left[2^{8}\right]: \mathrm{S}_{3}$ & $H .2$ & 5 & 2 \\
$\mathrm{G}_{2}(5)$ & 3906 & $5_{+}^{1+4}: \mathrm{GL}_{2}(5)$ & $H$ & 4 & 1 \\
& 3906 & $5^{2+3}: \mathrm{GL}_{2}(5)$ & $H$ & 4 & 1 \\
\hline $\mathrm{J}_{1}$ & 2926 & $\mathrm{~S}_{3} \times \mathrm{D}_{10}$ & $H$ & 67 & 1 \\
$\mathrm{HS}$ & 3850 & $2^{4} \cdot \mathrm{S}_{6}$ & $H: 2$ & 12 & 2 \\
$\mathrm{M}_{24}$ & 3795 & $2^{6}:\left(\mathrm{L}_{3}(2) \times \mathrm{S}_{3}\right)$ & $H$ & 5 & 1 \\
$\mathrm{Ru}$ & 4060 & ${ }^{2} \mathrm{~F}_{4}(2)$ & $H$ & 3 & 1 \\
$\mathrm{Fi}_{22}$ & 3510 & $2 . \mathrm{U}_{6}(2)$ & $H: 2$ & 3 & 2 \\
\hline
\end{tabular}

Table 7: Primitive diagonal type groups

\begin{tabular}{llll}
\hline $\begin{array}{l}\text { Primitive } \\
\text { group } G\end{array}$ & Degree & $\begin{array}{l}\text { Rank } \\
\text { of } N\end{array}$ & $\begin{array}{l}\text { Cohort } \\
\text { size }\end{array}$ \\
\hline $\mathrm{A}_{7}^{2}$ & 2520 & 8 & 5 \\
$\mathrm{~L}_{2}(19)^{2}$ & 3420 & 11 & 5 \\
$\mathrm{~A}_{5}^{3}$ & 3600 & 17 & 5 \\
$\mathrm{~L}_{2}(16)^{2}$ & 4080 & 7 & 8 \\
\hline
\end{tabular}


Table 8: Primitive product action groups

\begin{tabular}{lllll}
\hline Socle & Conditions & Degree & $\begin{array}{l}\text { Rank } \\
\text { of } N\end{array}$ & $\begin{array}{l}\text { Cohort } \\
\text { size }\end{array}$ \\
\hline $\mathrm{A}_{n}^{2}$ & $50 \leq n \leq 63$ & $n^{2}$ & 3 & 4 \\
$\mathrm{~L}_{2}(p)^{2}$ & $53 \leq p \leq 61$ & $(p+1)^{2}$ & 3 & 4 \\
\hline $\mathrm{L}_{2}(49)^{2}$ & & 2500 & 3 & 24 \\
$\mathrm{U}_{3}(5)^{2}$ & & 2500 & 6 & 4 \\
$\mathrm{~L}_{3}(3)^{2}$ & 2704 & 10 & 3 \\
$\mathrm{~L}_{2}(11)^{2}$ & 3025 & 21 & 4 \\
$\mathrm{~L}_{2}(11)^{2}$ & & 3025 & 21 & 3 \\
$\mathrm{M}_{11}^{2}$ & 3025 & 6 & 1 \\
$\mathrm{~A}_{11}^{2}$ & 3025 & 6 & 4 \\
$\mathrm{~A}_{8}^{2}$ & & 3136 & 10 & 4 \\
$\mathrm{~L}_{3}(4)^{2}$ & & 3136 & 6 & 24 \\
$\mathrm{~L}_{2}(19)^{2}$ & & 3249 & 10 & 1 \\
$\mathrm{~L}_{3}(7)^{2}$ & 3249 & 3 & 4 \\
$\mathrm{U}_{3}(3)^{2}$ & & 3969 & 10 & 4 \\
$\mathrm{U}_{3}(3)^{2}$ & & 3969 & 10 & 4 \\
$\mathrm{~S}_{6}(2)^{2}$ & 3969 & 6 & 1 \\
$\mathrm{~L}_{6}(2)^{2}$ & & 3969 & 3 & 1 \\
\hline$\left(\mathrm{A}_{5}^{2}\right)^{2}$ & & 3600 & 10 & 24 \\
\hline \hline $\mathrm{A}_{n}^{3}$ & $14 \leq n \leq 15$ & $n^{3}$ & 4 & 10 \\
\hline $\mathrm{L}_{2}(13)^{3}$ & & 2744 & 4 & 10 \\
$\mathrm{~A}_{6}^{3}$ & 3375 & 10 & 10 \\
$\mathrm{~A}_{7}^{3}$ & 3375 & 4 & 2 \\
$\mathrm{~A}_{8}^{3}$ & 3375 & 4 & 2 \\
\hline \hline $\mathrm{A}_{5}^{5}$ & 3125 & 6 & 26 \\
\hline & & & & \\
\hline
\end{tabular}




\section{References}

[1] M. Aschbacher, On the maximal subgroups of the finite classical groups, Invent. Math. 76(3) (1984) 469-514.

[2] E. R. Bennett, Primitive groups with a determination of the primitive groups of degree 20, Amer. J. Math. 34(1) (1912) 1-20.

[3] J. N. Bray, D. F. Holt, C. M. Roney-Dougal, The Maximal Subgroups of the Low-Dimensional Finite Classical Groups London Math. Soc. Lecture Notes, Cambridge Univeristy Press, to appear.

[4] W. Bosma, J. Cannon, C. Playoust, The Magma algebra system. I. The user language, J. Symbolic Comput. 24(3-4) (1997) 235-265.

[5] F. N. Cole, The transitive substitution-groups of nine letters, Bull. Amer. Math. Soc. 2(10) (1893) 250-259.

[6] J. H. Conway, R. T. Curtis, S. P. Norton, R. A. Parker, R. A. Wilson, Atlas of Finite Groups, Oxford University Press, Oxford, 1985.

[7] J. D. Dixon, B. Mortimer, The primitive permutation groups of degree less than 1000, Math. Proc. Cambridge Philos. Soc. 103(2) (1988) 213-238.

[8] J. D. Dixon, B. Mortimer. Permutation Groups, Graduate Texts in Mathematics vol. 163, Springer-Verlag, New York, 1996.

[9] B. Eick, B. Höfling. The solvable primitive permutation groups of degree at most 6560, London Math. Soc. J. Comput. Math. 6 (2003) 29-39.

[10] The GAP Group, GAP - Groups, Algorithms, and Programming, Version 4.4.10, 2007.

[11] G. Hiss, G. Malle, Low-dimensional representations of quasi-simple groups, London Math. Soc. J. Comput. Math. 5 (2002) 95-126. Corrigenda: London Math. Soc. J. Comput. Math. 4 (2001) 22-63.

[12] D. F. Holt, C. M. Roney-Dougal, Constructing maximal subgroups of classical groups, London Math. Soc. J. Comput. Math. 8 (2005) 46-79.

[13] D. F. Holt, C. M. Roney-Dougal, Constructing maximal subgroups of orthogonal groups, In preparation.

[14] B. Huppert, Endliche Gruppen. I, Die Grundlehren der Mathematischen Wissenschaften, Band 134, Springer-Verlag, Berlin, 1967.

[15] C. Jordan, Traité des substitutions et des équations algébriques, Les Grands Classiques Gauthier-Villars, Éditions Jacques Gabay, Sceaux, 1989. Reprint of the 1870 original. 
[16] P. Kleidman, M. Liebeck, The subgroup structure of the finite classical groups, London Math. Soc. Lecture Note Series vol. 129, Cambridge University Press, Cambridge, 1990.

[17] P. B. Kleidman, The maximal subgroups of the finite 8-dimensional orthogonal groups $P \Omega_{8}^{+}(q)$ and of their automorphism groups, J. Algebra 110(1) (1987) 173-242.

[18] P. B. Kleidman, R. A. Wilson, The maximal subgroups of $E_{6}(2)$ and Aut $\left(E_{6}(2)\right)$, Proc. London Math. Soc. (3) 60(2) (1990) 266-294.

[19] V. Landazuri, G. M. Seitz, On the minimal degrees of projective representations of the finite Chevalley groups, J. Algebra 32 (1974) 418-443.

[20] M. W. Liebeck, On the orders of maximal subgroups of the finite classical groups, Proc. London Math. Soc. (3) 50(3) (1985), 426-446.

[21] M. W. Liebeck, J. Saxl, Primitive permutation groups containing an element of large prime order, J. London Math. Soc. (2) 31(2) (1985) 237-249.

[22] F. Lübeck, Small degree representations of finite Chevalley groups in defining characteristic, London Math. Soc. J. Comput. Math. 4 (2001) 135-169.

[23] E. N. Martin, On the imprimitive substitution groups of degree fifteen and the primitive substitution groups of degree eighteen, Amer. J. Math. 23(3) (1901) 259-286.

[24] G. A. Miller, Note on the transitive substitution groups of degree twelve, Bull. Amer. Math. Soc. 1(10) (1895) 255-258.

[25] G. A. Miller, List of transitive substitution groups of degree twelve, Quart. J. Pure. Appl. Math. 28 (1896) 193-231.

[26] G. A. Miller, On the primitive substitution groups of degree fifteen, Proc. London Math. Soc. 28(1) (1897) 533-544.

[27] G. A. Miller, Sur l'énumeration des groupes primitifs dont the degré est inférieur à 17, C. R. Acad. Sci. 124 (1897) 1505-1508.

[28] G. A. Miller, On the primitive substitution groups of degree sixteen, Amer. J. Math. 20(3) (1898) 229-241.

[29] G. A. Miller, On the transitive substitution groups of degrees 13 and 14. Quart. J. Pure. Appl. Math. 29 (1898) 224-249.

[30] G. A. Miller, On the transitive substitution groups of degree seventeen, Quart. J. Pure. Appl. Math. 31 (1900) 49-57.

[31] S. Norton, Improvements to the ATLAS, http://brauer.maths.qmul.ac.uk/Atlas 
[32] C. M. Roney-Dougal, The primitive permutation groups of degree less than 2500, J. Algebra 292(1) (2005) 154-183.

[33] C. M. Roney-Dougal, W. R. Unger, The affine primitive permutation groups of degree less than 1000, J. Symbolic Comput. 35(4) (2003) 421-439.

[34] M. W. Short, The primitive soluble permutation groups of degree less than 256, Lecture Notes in Mathematics vol. 1519, Springer-Verlag, Berlin, 1992.

[35] C. C. Sims, Computational methods in the study of permutation groups, in: Computational Problems in Abstract Algebra (Proc. Conf., Oxford, 1967), Pergamon, Oxford, 1970, pp. 169-183.

[36] M. Suzuki, On a class of doubly transitive groups, Ann. of Math. (2) 75 (1962) 105-145.

[37] H. Theißen, Eine Methode zur Normalisatorberechnung in Permutationsgruppen mit Anwendungen in der Konstruktion primitiver Gruppen, $\mathrm{PhD}$ thesis, RWTH, Aachen, Germany, 1997.

[38] R. Wilson, P. Walsh, J. Tripp, I. Suleiman, S. Rogers, R. Parker, S. Norton, S. Nickerson, S. Linton, J. Bray, R. Abbott, ATLAS of Finite Group Representations, http://web.mat.bham.ac.uk/atlas/v2.0/ 